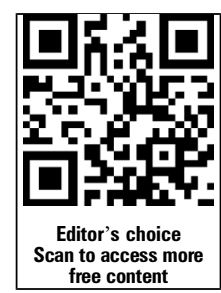

'Department of Neonatology, The National Maternity Hospital, Dublin, Ireland ${ }^{2}$ National Children's Research ${ }^{3}$ School of Medicine \& Medical Science, University College Dublin, Dublin, Ireland ${ }^{4}$ Department of Radiology, The National Maternity Hospital, Dublin, Ireland

\section{Correspondence to} Dr Emily A Kieran, Department of Neonatology, The National Maternity Hospital, Holles Street, Dublin 2, Ireland; emilykieran@gmail.com

Received 6 October 2014 Revised 4 July 2015 Accepted 13 July 2015 Published Online First 11 August 2015

\title{
Estimating umbilical catheter insertion depth in newborns using weight or body measurement: a randomised trial
}

\author{
Emily A Kieran, ${ }^{1,2,3}$ Eoghan E Laffan, ${ }^{4}$ Colm P F O'Donnell ${ }^{1,2,3}$
} Centre, Dublin, Ireland

\section{ABSTRACT}

Objective Incorrectly positioned umbilical venous and arterial catheters (UVC and UAC) are associated with increased rates of complications in newborns. Catheter insertion depth is often estimated using body surface measurement. We wished to determine whether estimating insertion depth of umbilical catheters using birth weight (BW), rather than surface measurements, results in more correctly positioned catheters.

Interventions/outcome Newborns were randomised to have UVC and UAC insertion depth estimated using formulae based on BW or using graphs based on shoulder-umbilicus length. The primary outcome was correct catheter tip position on X-ray determined by one radiologist masked to group assignment.

Results UVC insertion was successful in 97/101 (96\%) infants but the catheter was not advanced to the estimated depth in 22. There was no difference in the proportion of correctly positioned UVCs between groups (weight 16/51 (31\%) vs measurement $13 / 46(28 \%), p=0.826)$. The tips of $52(54 \%)$ UVCs were in the portal venous system or too low on X-ray. Attempted UAC insertion was successful in $62 / 87(71 \%)$ infants. More infants in the weight group had a correctly positioned UAC tip (weight 29/32 (91\%) vs measurement 15/30 (50\%), $p=0.001)$.

Conclusions UVCs were often not inserted to the estimated depth, and their tips were in the portal venous system or too low on X-ray. Using BW to estimate insertion depth did not result in more correctly positioned UVCS.

UAC insertion attempts were often unsuccessful, but when successful, using BW to estimate insertion depth resulted in more correctly positioned catheters.

Trial registration number (ISRCTN17864069).

\section{INTRODUCTION}

Umbilical vessel catheterisation of newborns was described more than 60 years ago ${ }^{1-3}$ and is still frequently performed. When umbilical catheters are inserted, they are secured in place and an anteroposterior X-ray is taken to determine the position of the catheter tip (figure 1). While umbilical venous catheters (UVCs) and umbilical arterial catheters (UACs) may be of benefit to infants, complications with their use have been reported. ${ }^{1}{ }^{4-6}$ CrossMark

To cite: Kieran EA, Laffan $\mathrm{EE}, \mathrm{O}^{\prime}$ Donnell CPF Arch Dis Child Fetal Neonatal Ed 2016;101: F10-F15.
The risk of complications is increased if the catheter tip is incorrectly positioned. As pericardial effusions and cardiac tamponade have been reported with UVCs whose tips lay within the heart, ${ }^{5-9}$ it is recommended to position UVC tips outside the heart at the junction of the right atrium and inferior vena cava. ${ }^{9} 10$ This position corresponds to the tip being visible between the upper

\section{What is already known on this topic}

Umbilical catheters are commonly inserted in newborns for monitoring and for fluid and drug administration.

- Fewer complications arise from correctly sited catheters.

- Insertion depth may be estimated using infant birth weight or body measurements.

\section{What this study adds}

Estimating insertion depth using a birth weight-derived formula resulted in more correctly placed umbilical arterial catheters.

- Umbilical venous catheters were frequently incorrectly placed whether insertion depth was estimated using weight or measurements.

border of the 9 th and the lower border of the 10th thoracic vertebrae (T9-T10) on X-ray (figure 1). ${ }^{10}$ It is recommended that UAC tips should lie in a high position ${ }^{11}$-that is, in the descending aorta above the level of the diaphragm and below the left subclavian artery, with the catheter tip visible between the upper border of $\mathrm{T} 6$ and the lower border of T10 on X-ray (figure 1). ${ }^{3}{ }^{10}$ Review of $\mathrm{X}$-rays showed that the tips were not in the recommended position in half of infants who had umbilical catheters inserted in our hospital.

UVCs and UACs are marked at $1 \mathrm{~cm}$ intervals from the tip so that the depth to which they are inserted is known. Several methods of estimating an appropriate insertion depth for umbilical catheters have been described. ${ }^{12-19}$ Two methods that may be used to estimate insertion depths for both UVCs and UACs are widely used in clinical practice. ${ }^{12}{ }^{13}$ From a postmortem study of 50 infants, Dunn published graphs that related an infant's shoulder-umbilicus length to the insertion depth for UVCs and UACs (figures 2 and 3). ${ }^{12}$ Shukla and Ferrara $^{13}$ retrospectively studied 10 infants who had UVCs inserted and 43 infants who had UACs inserted. He found that birth weight (BW) correlated better with catheter insertion depth than three body surface measurements that included Dunn's shoulder-umbilicus length and published mathematical formulae to estimate UVC and UAC insertion depth based on BW. ${ }^{13}$ In a retrospective 


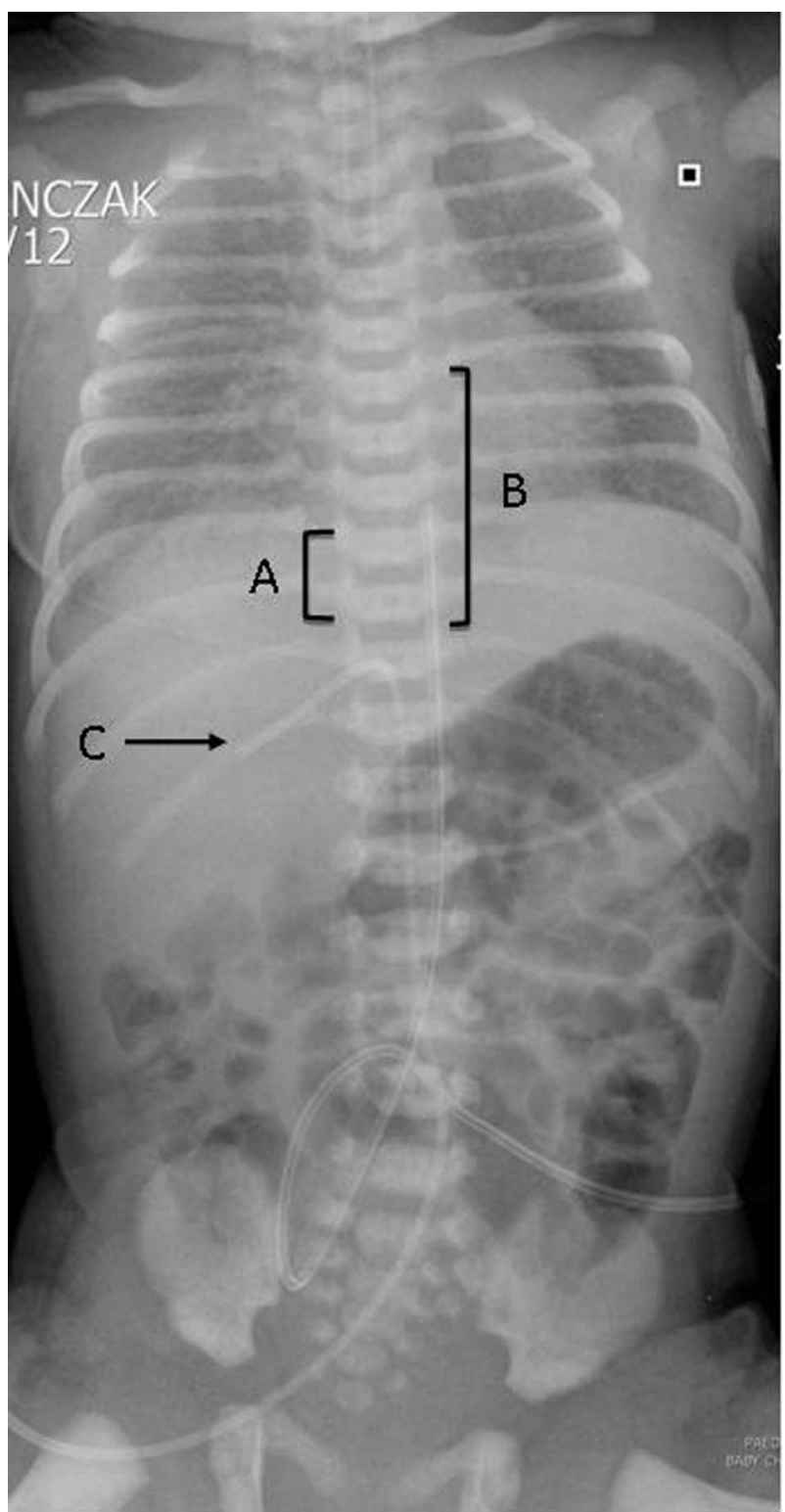

Figure 1 Anteroposterior X-ray showing correct catheter tip placement for (A) umbilical venous catheter (UVC) - between upper border of 9th and lower border of 10th thoracic vertebrae, and (B) umbilical arterial catheter (UAC) - between upper border of 6th and lower border of 10 th thoracic vertebrae and $(C)$ tip in portal circulation.

cohort study, Verheij et al found that the Dunn method was more accurate for predicting UVC insertion depth and the Shukla method was more accurate for predicting UAC insertion depth. ${ }^{20}$ We wished to prospectively compare the Dunn and Shukla methods of estimating insertion depths for UVCs and UACs in term and preterm infants in a randomised fashion.

\section{PATIENTS AND METHODS}

We conducted this randomised controlled trial at the National Maternity Hospital (NMH), Dublin, a stand-alone university maternity hospital with a level 3 neonatal intensive care unit (NICU) and approximately 9500 deliveries annually. All infants are weighed on electronic scales on admission to the NICU. Prior to this study, umbilical catheter insertion depths were usually estimated using shoulder-umbilicus length. Term and preterm infants without congenital anomalies were eligible for

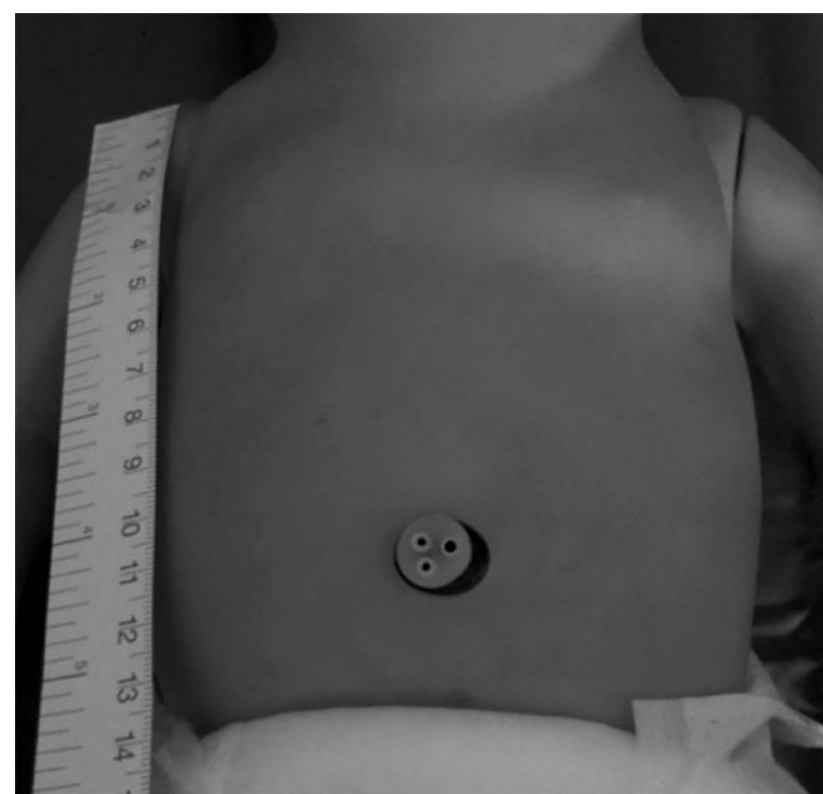

Figure 2 Shoulder-umbilicus length-the distance measured in an inferior vertical direction from the shoulder tip to the level of the umbilicus—as measured by Dunn. ${ }^{12}$

inclusion if they were undergoing umbilical vessel catheterisation in the NICU. The study protocol was approved by the Ethics Committee at the NMH and was registered with Current Controlled Trials (http://www.controlled-trials.com/ ISRCTN17864069/WORM).

If it was anticipated that an infant would undergo umbilical vessel catheterisation following delivery (eg, in the case of a planned extremely preterm delivery), written informed consent was obtained prior to enrolment in the study from a parent or guardian by a member of the research team. If umbilical vessel catheterisation was performed as an emergency procedure, for example, a critically ill term infant born unexpectedly in poor condition and requiring UVC/UAC insertion on admission to the NICU, we had ethical approval to enrol such infants using a waiver of informed consent. In such situations infants were enrolled at the time of UVC/UAC insertion and a member of the research team informed parents as soon as possible that their infant had been enrolled in the study, provided them with written information about the study and sought written consent for their infant's information to be collected and included in analysis.

We generated the randomisation schedule in blocks of four using a random number table and kept it concealed from treating clinicians. The randomisation was stratified by BW $(<1500$ or $\geq 1500 \mathrm{~g}$ ). Infants were randomised in a $1: 1$ ratio to have their umbilical catheter insertion depth estimated using 'weight' or 'measurement' and infants of multiple pregnancies were randomised as individuals. The group assignments were printed on cards that were folded and placed in sequentially numbered, sealed, opaque envelopes. The envelopes were opened just before UVC and/or UAC insertion was attempted in the NICU.

\section{WEIGHT GROUP}

Infants had the umbilical catheter insertion depth estimated using the formulae described by Shukla and Ferrara, ${ }^{13}$ that is:

- UVC insertion depth $(\mathrm{cm})=(\mathrm{BW} \times 1.5)+5$

- UAC insertion depth $(\mathrm{cm})=(\mathrm{BW} \times 3)+9$ 
Figure 3 Graphs relating measurement of the shoulder-umbilicus length to the estimated insertion depth for umbilical venous catheters (UVCs) (left) and umbilical arterial catheters (UACs) (right). ${ }^{12}$ On both graphs, the red lines indicate the depth that estimates insertion to the diaphragm that were used in the study. Reproduced with kind permission from Dunn PM.

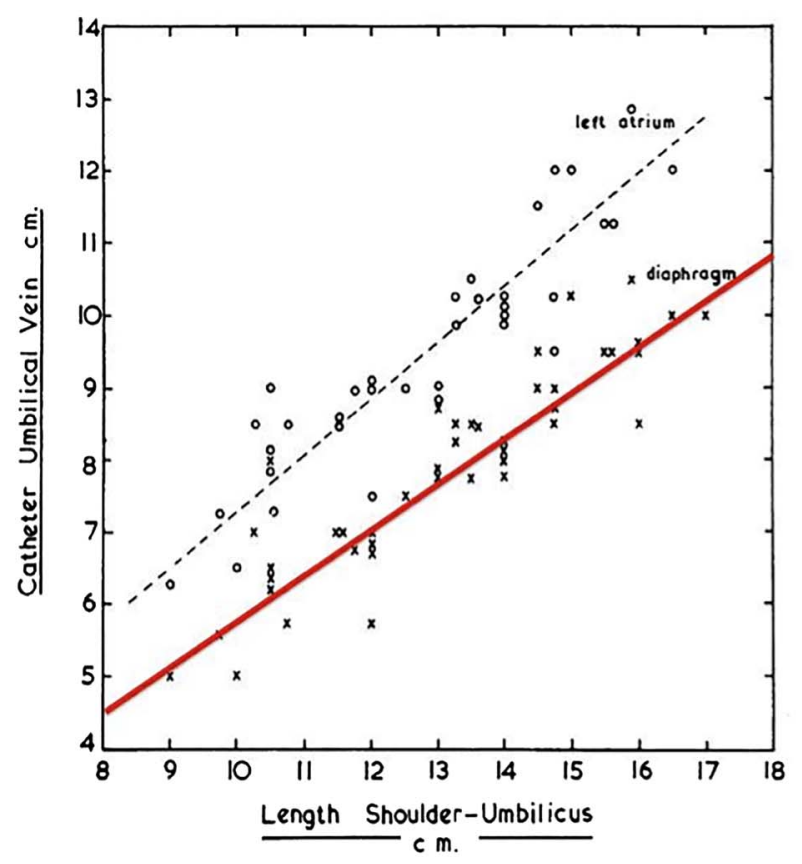

These formulae were printed on the card indicating assignment to the weight group. Clinicians filled in the infants' BW into the preprinted formula, calculated the insertion depth and recorded it on the card.

\section{MEASUREMENT GROUP}

Clinicians measured the vertical distance from the infants' shoulder tip to the level of the umbilicus (figure 2). They then applied this measurement to copies of the graphs described by Dunn (figure 3) to estimate the insertion depth ${ }^{12}$ that were kept in the NICU specifically for this purpose. The clinicians recorded both the shoulder-umbilicus length and the resulting estimated insertion depth it yielded in a space provided on the card indicating assignment to the measurement group.

The decision to insert umbilical catheters was at the discretion of the attending clinician. UVCs and UACs were inserted under sterile conditions following local clinical guidelines. Size 4-French double lumen radio-opaque polyurethane UVCs and single-lumen UACs (size 3.5 or 5 French) were inserted (all Vygon, Ecouen, France). Infants were placed supine on a mattress while the umbilical catheters were inserted. Clinicians were advised to advance the catheters until the marker that indicated the estimated insertion depth was at the level of the skin on the abdominal wall, to confirm that blood could be aspirated through the catheter with a syringe and to then suture the catheter in place. If the catheter could not be advanced to the estimated depth or blood could not be aspirated, the catheter was withdrawn to a position where blood could be aspirated. The clinician who inserted the catheter recorded the depth at which they secured it on the group assignment card. Infants remained supine until an anteroposterior X-ray of the infant's chest and abdomen was taken by a radiographer who was masked to group assignment (figure 1). Radio-opaque contrast medium was not injected into the lines before or during the X-ray. This X-ray was used by clinicians to confirm line tip position and catheter position was adjusted at their discretion. The X-ray was also used to determine the primary outcome of this study.

The primary outcome for our study was correct placement of the umbilical catheters. Correct placement of the UVC was defined as the catheter tip being visible between T9 and T10 on $\mathrm{X}$-ray. Correct placement for UAC was defined as the catheter tip being visible between T6 and T10 on X-ray (figure 1). The position of the catheter tips was determined by one consultant radiologist (EEL) who was masked to the infants' group assignment. We considered UVC and UAC separately and both catheters did not have to be correctly placed for an infant to reach the primary outcome. We recorded clinically relevant secondary outcomes and possible adverse events related to the placement and use of UVCs and UACs. We recorded the duration that the umbilical catheters were in situ and the duration of hospital stay from case notes. Necrotising enterocolitis was diagnosed if the criteria of Bell stage 2 were fulfilled (pneumatosis and/or perforation on X-ray). Blood culture-positive sepsis was diagnosed when a pathogen (eg, Staphylococcus aureus) was present in a single blood culture or when potential contaminants (eg, coagulase-negative staphylococci) were grown on two separate blood cultures drawn when an indwelling line was in situ or within $24 \mathrm{~h}$ of its removal.

\section{SAMPLE SIZE ESTIMATION}

We estimated that we would need to enrol 92 infants to show a reduction in incorrect catheter tip placement from 55\% with insertion depth estimation using the shoulder-umbilicus length to $25 \%$ by using the BW formula with a two-tailed type I error rate of 0.05 and a power of $80 \%$. We estimated that $10 \%$ of attempts at umbilical catheterisation would be unsuccessful; to compensate, we aimed to enrol 101 infants. Data were analysed using the intention-to-treat principle with PASW V.20 software (IBM, Armonk, New York, USA). We compared the primary outcome and dichotomous secondary outcomes with nonparametric tests (Fisher's exact test), continuous secondary outcomes with parametric tests ( $t$ test) and considered $p$ values $<0.05$ statistically significant.

\section{RESULTS}

One hundred and twenty-one infants underwent umbilical vessel catheterisation in the NICU of the NMH between July 2012 and July 2013. Fourteen infants were not enrolled into 


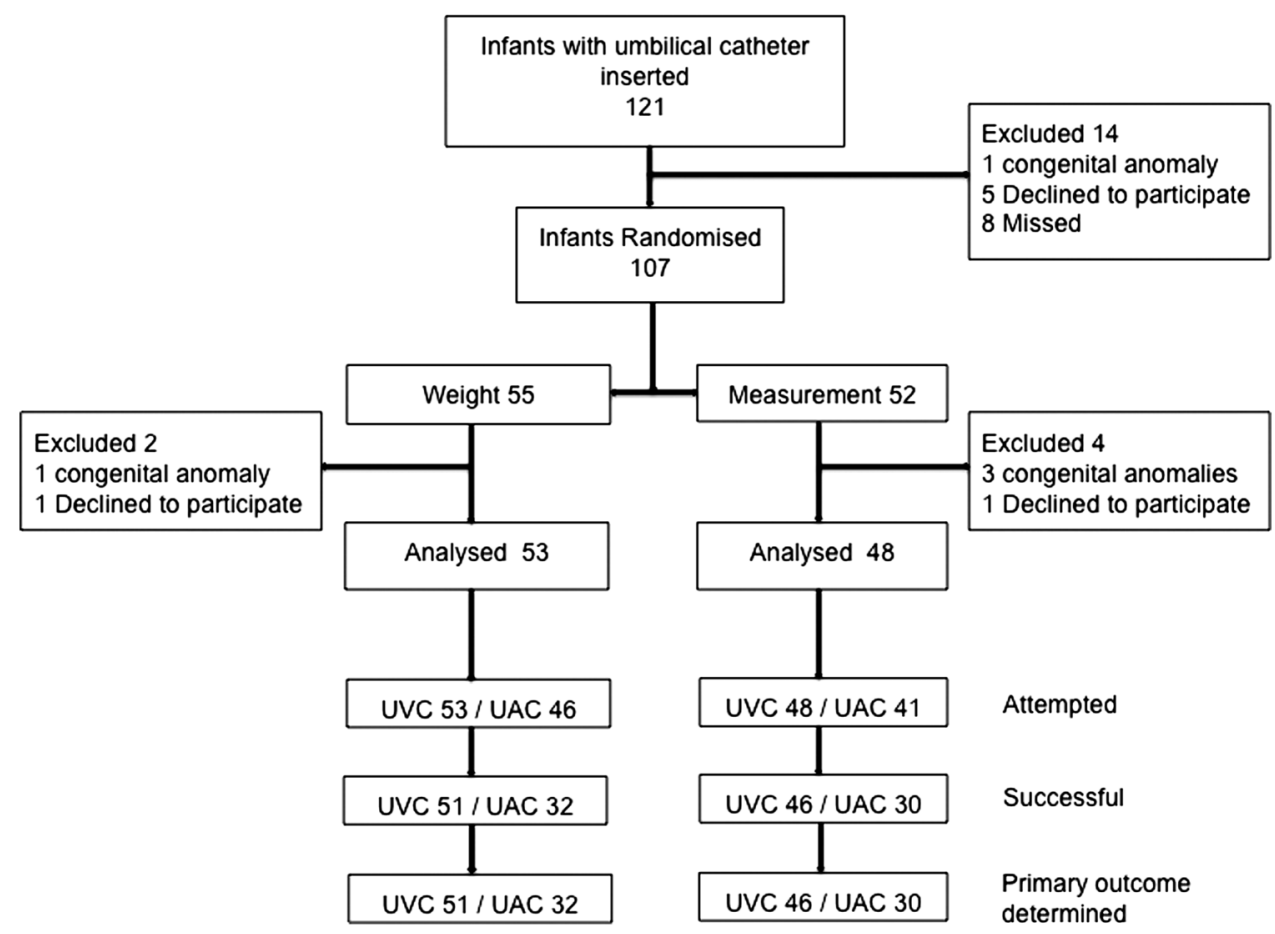

Figure 4 Patient recruitment. UAC, umbilical arterial catheter; UVC, umbilical venous catheter.

the study (figure 4). Six of the 107 infants who were randomised were later excluded (figure 4). Parental consent was obtained prior to randomisation for 52 infants, all of whom were $<31$ weeks gestation at delivery. Forty-nine infants were enrolled using the waiver of informed consent with written parental consent obtained soon after inclusion. We analysed data for 101 infants, of whom 53 were randomised to weight and 48 to measurement. The groups were well matched for gestational age, BW, gender and respiratory support at time of randomisation (table 1).

Thirteen different clinicians inserted umbilical catheters, the majority (91\%) were inserted by nine registrars (trainees with 6 months to 2 years experience in neonatology); the remainder were inserted by three consultant neonatologists $(8 \%)$ and one advanced neonatal nurse practitioner (1\%).

UVC insertion was attempted in 101 infants and was successful in 97 (96\%). There was no difference in the proportion of correctly placed UVCs between the two groups (weight 16/51 (31\%) vs measurement $13 / 46(28 \%) \mathrm{p}=0.826)$ (table 2).

The UVC was secured at less than the estimated insertion depth in 22/97 (23\%) infants. The reasons for underinsertion

\begin{tabular}{|c|c|c|}
\hline & Weight $(\mathrm{N}=53)$ & Measurement $(\mathrm{N}=48)$ \\
\hline Gestational age (weeks)* & $29(27,39)$ & $29(26,39)$ \\
\hline$B W(g)^{*}$ & $1250(910,3065)$ & $1313(856,3563)$ \\
\hline $\mathrm{BW}<1500 \mathrm{~g} \dagger$ & $31(58)$ & $28(58)$ \\
\hline Malet & $24(45)$ & $24(50)$ \\
\hline Ventilated at randomisationt & $34(64)$ & $27(56)$ \\
\hline Nasal CPAP at randomisationt & $12(23)$ & $15(31)$ \\
\hline
\end{tabular}

were that the operator could not insert the catheter to the estimated depth in 15 infants, the operator could not aspirate blood from the catheter in 5 infants and values were not recorded for 2 infants. Of the UVCs that were secured at the estimated depth, fewer catheters in the weight group had the tip in a low position (ie, below T10) on X-ray (weight 4/38 (11\%) vs measurement 10/35 (29\%), $\mathrm{p}=0.074)$. Overall, $30 \quad(31 \%)$ UVCs were in a low position and $22(23 \%)$ UVCs were in the portal circulation on X-ray (figure 1). Analysing infants whose UVC was secured at the correct depth and was not in the liver, there was no difference in the primary outcome between groups (weight 16/30 (44\%) vs measurement 12/27 (44\%), p=0.599).

UAC insertion was attempted in 87 infants and was successful in $62(71 \%)$ (table 3).

Most infants in the weight group had the UAC tip placed correctly compared with half of infants in the measurement group (29/32 (91\%) vs $15 / 30(50 \%) \mathrm{p}=0.001)$. No infant in the weight group had a UAC tip below T10 on X-ray. The UAC tip was too low (below T10) in all infants in the measurement group who had incorrectly placed UACs.

\begin{tabular}{lccl} 
Table 2 & Outcomes for UVCs \\
\hline UVC & Weight & Measurement & p Value \\
\hline Successfully inserted & $51 / 53(96)$ & $46 / 48(96)$ & 1.0 \\
Secured at estimated depth & $38 / 51(75)$ & $35 / 46(76)$ & 1.0 \\
Secured at more than estimated depth & $2 / 51(4)$ & $0 / 46(0)$ & 0.496 \\
Secured at less than estimated depth & $11 / 51(22)$ & $11 / 46(24)$ & 0.812 \\
Primary outcome--tip T9-T10 & $16 / 51(31)$ & $13 / 46(28)$ & 0.826 \\
Tip above T9 & $11 / 51(22)$ & $5 / 46(11)$ & 0.181 \\
Tip below T10 & $10 / 51(20)$ & $20 / 46(43)$ & 0.015 \\
Tip in portal circulation & $14 / 51(27)$ & $8 / 46(17)$ & 0.332 \\
\hline Data are $n$ (\%) compared with Fisher's exact test. & & \\
UVC, umbilical venous catheter. & &
\end{tabular}




\begin{tabular}{lccl} 
Table 3 & Outcomes for UACs \\
\hline UAC & Weight & Measurement & p Value \\
\hline Successfully inserted & $32 / 46(70)$ & $30 / 41(73)$ & 0.915 \\
Secured at estimated depth & $29 / 32(91)$ & $28 / 30(93)$ & 1.0 \\
Secured at more than estimated depth & $2 / 32(6)$ & $2 / 30(7)$ & 1.0 \\
Secured at less than estimated depth & $1 / 32(3)$ & $0 / 30(0)$ & 1.0 \\
Primary outcome-tip T6-T10 & $29 / 32(91)$ & $15 / 30(50)$ & 0.001 \\
Tip above T6 & $3 / 32(9)$ & $0 / 30(0)$ & 0.238 \\
Tip below T10 & $0 / 32(0)$ & $15 / 30(50)$ & 0.000 \\
\hline Data are $n$ (\%) compared with Fisher's exact test. & & \\
UAC, umbilical arterial catheter. & & &
\end{tabular}

There was no difference in any of the recorded secondary outcomes between the two groups (table 4).

One infant in the measurement group had the UAC removed because of concerns of vascular compromise to the lower limbs. There were no confirmed episodes of umbilical catheter-related thrombosis.

\section{DISCUSSION}

In contrast to a previous cohort study, ${ }^{20}$ we found no difference in the rate of correctly positioned UVC whether insertion depth was estimated using weight or body measurement. A striking feature of our study is the high rate of UVCs that were in the portal circulation on X-ray. We speculate that the large number of UVCs that were not inserted to the estimated insertion depth could not be advanced because they also entered the portal circulation at the time of insertion. We anticipated that a proportion of UVCs would not pass through the ductus venosus into the inferior vena cava, but were surprised to confirm this in $23 \%$ of cases and suspect that it happened in about half of attempts. Techniques have been reported for decreasing the chance of portal placement of the UVC, such as a double-catheter technique ${ }^{2122}$ or external liver mobilisation. ${ }^{23} \mathrm{~A}$ technique that can be used at the time of UVC insertion that reduces the incidence of UVC placement in the liver is highly desirable.

In keeping with previous cohort studies, ${ }^{13}{ }^{20}$ we found that estimating the insertion depth of UACs in newborn infants using weight rather than body surface measurement resulted in more correctly positioned catheters. Though the rate of failure of attempted umbilical artery catheterisation rate in our study was disappointingly high (29\%), it is comparable with that seen in previous studies. ${ }^{20}$

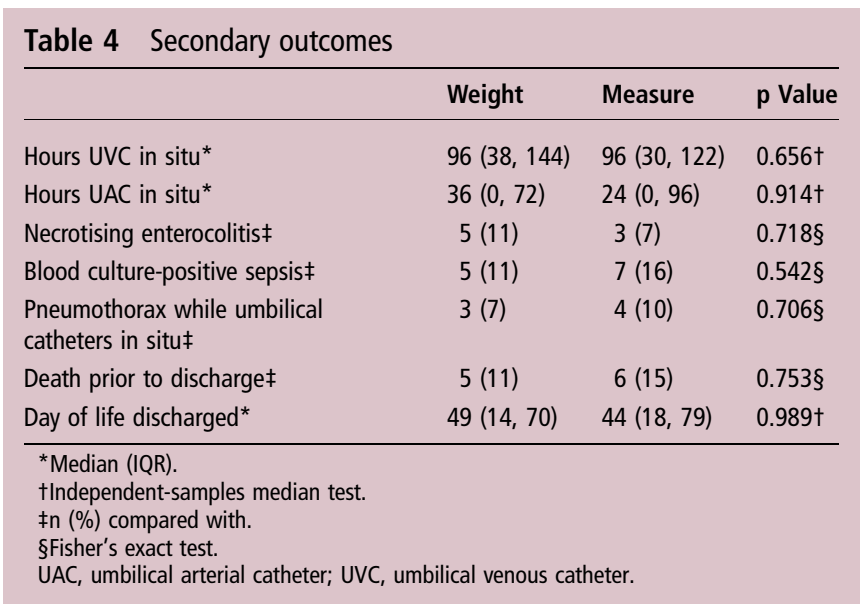

One strength of our study is that the primary outcome was determined by one radiologist who was unaware of group assignment and unaware whether or not the catheters were secured at the estimated depth. We used X-ray to determine the primary outcome of our study, as this is the method most commonly used to assess umbilical catheter tip position in clinical practice, both in our unit and worldwide. X-ray was the method used to determine tip position in the majority of previous studies and it is the method most often recommended in guidelines. ${ }^{9} 1518{ }^{20}$ While ultrasound has been used to and may be superior for determining umbilical catheter tip position, ${ }^{24-28}$ we do not have routine immediate access to this method of determination in clinical practice.

A weakness of our study is the small number of infants enrolled; however, this number exceeds that enrolled in most previous studies. Also, the operators inserting the UVCs and UACs were not masked to the infants' group assignment. The marks on the catheters that we used are often inaccurate. ${ }^{29}$ The differences between the indicated and actual distance from the tip are small; however, they may be clinically important, particularly in infants who have 3.5-French UACs inserted. As the model of catheter was the same in all enrolled infants, we believe that randomisation should have balanced any effect of inaccuracies in markings between the groups. While the shoulder-umbilicus length is frequently used to estimate the insertion depth of umbilical catheters, it appears that many clinicians do so incorrectly. A survey of 101 paediatric doctors, including 45 consultant paediatricians, showed that only 14/101 $(14 \%)$ used the measurement described by Dunn correctly. ${ }^{30}$ Our results should be extrapolated with caution if the shoulderumbilicus length is measured incorrectly.

\section{CONCLUSION}

Estimating UVC insertion depth using weight did not result in more correctly placed UVCs. UVCs were often not advanced to the estimated insertion depth or the catheter tip was in the portal venous system on X-ray. Attempted UAC insertion was often unsuccessful; when successful, estimating insertion depth using weight resulted in more correctly placed catheters.

Contributors EAK made substantial contributions to the conception and design of the study. She acquired the data and made a substantial contribution to the analysis and interpretation of the data. She wrote the first draft of the manuscript. EEL made substantial contributions to the design of the study. He determined the primary outcome of the study for each infant enrolled. He critically revised the manuscript for important intellectual content. CPFO conceived and designed the study. He analysed and interpreted the data. He redrafted the manuscript and revised it for important intellectual content. All authors approve this version of the manuscript.

Funding The investigators were supported by the National Children's Research Centre.

Competing interests None declared.

Ethics approval Research and Ethics committee, The National Maternity Hospital, Holles Street, Dublin 2, Ireland.

Provenance and peer review Not commissioned; externally peer reviewed.

\section{REFERENCES}

1 Cochran WD, Davis HT, Smith CA. Advantages and complications of umbilical artery catheterization in the newborn. Pediatrics 1968;42:769-77.

2 Gupta JM, Roberton NRC, Wigglesworth JS. Umbilical artery catheterization in the newborn. Arch Dis Child 1968:43:382-7.

3 Egan EA, Eitzman DV. Umbilical vessel catheterization. Am J Dis Child 1971;121:213-18.

4 Scott JM. latrogenic lesions in babies following umbilical vein catheterization. Arch Dis Child 1965;40:426-9.

5 Kulkarni PB, Dorard RD. Hydrothorax: a complication of intracardiac placement of umbilical venous catheter. J Pediatr 1979;94:813-15. 
6 Purohit DM, Levkoff AH. Pericardial effusion complicating umbilical venous catheterization. Arch Dis Child 1977;52:520.

7 Darling JC, Newell SJ, Dear PRF. Placement of neonatal central venous catheter tips in the right atrium: a practice to be avoided? Arch Dis Child Fetal Neonatal Ed 2001;85:F146.

8 Darling JC, Newell SJ, Mohamdee 0, et al. Central venous catheter tip in the right atrium: a risk factor for neonatal cardiac tamponade. J Perinatology 2001:21:461-4.

9 Department of Health (UK). Review of the deaths of four babies due to cardiac tamponade associated with the presence of central venous catheter. 2001. https://webarchive.nationalarchives.gov.uk/+/www.dh.gov.uk/en/ publicationsandstatistics/Publications/PublicationsPolicyAndGuidance/DH_4082465 (accessed 1 Feb 2012 and 28 Jan 2014).

10 Gomella TL, Cunningham MD, Eyal FG, et al. Neonatology, management, procedures, on-call problems, diseases and drugs. 5th edn. Lange Medical books/ McGraw-Hill, 2005.

11 Barrington KJ. Umbilical artery catheters in the newborn: effects of position of the catheter tip. Cochrane Database Syst Rev 2000;(2):CD000505. doi:10.1002/ 14651858.CD000505

12 Dunn PM. Localization of the umbilical catheter by post-mortem measurement. Arch Dis Child 1966:41:69-75.

13 Shukla H, Ferrara A. Rapid estimation of insertional length of umbilical catheters in newborns. Am J Dis Child 1986;140:786-8.

14 Vali P, Fleming SE, Kim JH. Determination of umbilical catheter placement using anatomic landmarks. Neonatology 2010;98:381-6.

15 Verheij GH, te Pas AB, Smits-Wintjens VEHJ, et al. Revised formula to determine the insertion length of umbilical vein catheters. Eur J Pediatr 2013;172:1011-15.

16 Rubin BK, McRobert E, O'Neill MB. An alternate technique to determine umbilical arterial catheter length. Clin Pediatr 1986;25:407-8.

17 Wright IMR, Owers M, Wagner MN. The umbilical arterial catheter: a formula for improved positioning in the very low birth weight infant. Pediatr Crit Care Med 2008:9:498-501
18 Sritipsukho S, Sritipsukho P. Simple and accurate formula to estimate umbilical arterial catheter length of high placement. J Med Assoc Thai 2007;90:1793-7.

19 Kumar PP, Kumar CD, Nayak M, et al. Umbilical arterial catheter insertion length: in quest of a universal formula. J Perinatol 2012;32:604-7.

20 Verheij GH, te Pas AB, Witlox RSGM, et al. Poor accuracy of methods currently used to determine umbilical catheter insertion length. Int J Pediatr 2010;2010:873167.

21 Davies MW, Cartwright DW. Insertion of umbilical venous catheters past the ductus venosus using the double catheter technique. Arch Dis Child Fetal Neonatal Ed 1998;78:F234.

22 Mandel D, Mimouni FB, Littner $Y$, et al. Double catheter technique for misdirected umbilical vein catheter. J Pediatr 2001;139:591-2.

23 Pennyforte $T$, Klosowski $S$, Alexandre $C$, et al. Increased success rate in umbilical venous catheter positioning by posterior liver mobilization. Arch Pediatr 2010;17:1440-4

24 Simanovsky N, Ofek-Shlomai N, Rozovsky K, et al. Umbilical venous catheter position: evaluation by ultrasound. Eur Radiol 2011;21:1882-6.

25 Greenberg M, Movahed H, Peterson B, et al. Placement of umbilical venous catheters with use of bedside real time ultrasonography. J Pediatr 1995; 126:633-5

26 Ades A, Sable C, Cummings $S$, et al. Echocardiographic evaluation of umbilical venous catheter placement. J Perinatol 2003;23:24-8.

27 Michel F, Brevaut-Malaty V, Pasquali R, et al. Comparison of ultrasound and X-ray in determining the position of umbilical venous catheters. Resuscitation 2012;83:705-9

28 Hoellering $A B$, Koorts PJ, Cartwright DW, et al. Determination of umbilical venous catheter tip position with radiograph. Pediatr Crit Care Med 2014;15:56-61.

29 Kieran EA, O'Donnell CPF. Accuracy of length measurements on neonatal umbilical catheters. Arch Dis Child Fetal Neonatal Ed 2014;99:F520-21.

30 Lopriore E, Verheij GH, Walter FJ. Measurement of the 'shoulder-umbilical' distance for insertion of umbilical catheters in newborn babies: questionnaire study. Neonatology 2008;94:34-7. 